\title{
Gradhiva
}

\section{Adam Jones, Peter Sebald (éd.), An African Family Archive. The Lawsons of Little Popo/Aneho (Togo),}

1841-1938

(«Sources of African History », vol. 7), Oxford, Oxford University Press, $2005,566 \mathrm{p}$.

\section{Olivier Pétré-Grenouilleau}

\section{OpenEdition}

\section{Journals}

Édition électronique

URL : http://journals.openedition.org/gradhiva/1242

DOI : $10.4000 /$ gradhiva. 1242

ISSN : 1760-849X

\section{Éditeur}

Musée du quai Branly Jacques Chirac

Édition imprimée

Date de publication : 15 novembre 2008

Pagination : 151-153

ISBN : 978-2-915133-94-3

ISSN : 0764-8928

\section{Référence électronique}

Olivier Pétré-Grenouilleau, « Adam Jones, Peter Sebald (éd.), An African Family Archive. The Lawsons of Little Popo/Aneho (Togo), 1847-7938 », Gradhiva [En ligne], 8 | 2008, mis en ligne le 28 octobre 2008, consulté le 21 septembre 2020. URL : http://journals.openedition.org/gradhiva/1242 ; DOI : https:// doi.org/10.4000/gradhiva.1242

Ce document a été généré automatiquement le 21 septembre 2020

(c) musée du quai Branly 


\section{Adam Jones, Peter Sebald (éd.), An African Family Archive. The Lawsons of Little Popo/Aneho (Togo), 1841-1938}

(«Sources of African History », vol. 7), Oxford, Oxford University Press, 2005, 566 p.

Olivier Pétré-Grenouilleau

\section{RÉFÉRENCE}

Adam Jones, Peter Sebald (éd.), An African Family Archive. The Lawsons of Little Popo/Aneho (Togo), 1841-1938(« Sources of African History », vol. 7), Oxford, Oxford University Press, 2005, 566 p.

Le présent ouvrage a pour objet la publication du Grand Livre Lolamé, dans lequel sont consignées les lettres officiellement adressées ou envoyées par les Lawsons, une importante famille africaine de Little Popo (Aneho depuis 1905), sur la côte de l'actuel Togo. Mais ses auteurs, Adam Jones et Peter Sebald, nous livrent ici beaucoup plus que le fruit d'un travail d'éditeurs. Pour deux raisons. La première est que ce livre résulte d'un formidable travail de chercheurs. C'est en effet dans les années 1975-1977 que Nicoué Gayibor découvrait véritablement l'intérêt historique et historiographique du Grand Livre Lolamé (volume de $52 \times 35 \times 6 \mathrm{~cm}$ pesant 7,5 kg) dont l'accès direct nous est ici permis. Mais il fallut une dizaine d'années (1995-2005) pour que nos deux "éditeurs » réussissent à le publier dans son intégralité (ce qui, notent-ils, est sans doute la première tentative en la matière, beaucoup d'autres documents familiaux africains n'étant accessibles qu'en partie). Il leur a fallu pour cela se faire accepter, passer par une première transcription sur une simple machine à écrire, avant de pouvoir photocopier l'ensemble et de le convertir en fichiers informatisés. Sans oublier, évidemment, l'important travail sur la source proprement dite, afin de la rendre 
accessible : essayer de combler les passages manquants, ajouter des notes, classer le tout en chapitres, etc.

Les 719 documents contenus dans cette source sont ici présentés en sept parties chronologiques (1841-1853, 1854-1877, 1880-1883, 1884, 1885-1887, 1888-1973 et documents non datés dont de très intéressants textes de nature historiographique sur l'histoire du Dahomey ou bien celle du célèbre Francisco de Souza), d'importance inégale d'un point de vue purement documentaire (200 documents pour la période 1841-1849 par exemple, pour seulement 17 entre 1888 et 1973). Le tout est complété par des annexes et index d'un grand intérêt (chronologie, quartiers et "villes " de Little Popo, ancêtres des Lawsons...; index des lieux, navires et personnes - 1400 sont mentionnées dans le Grand Livre). L'ensemble constitue indéniablement un très important instrument de travail qui rendra d'éminents services à de nombreux chercheurs, qu'ils s'intéressent aux Lawsons, à Little Popo, ou encore à l'histoire du commerce et de la colonisation dans la région; second motif pour lequel il incombe véritablement de féliciter Adam Jones et Peter Sebald.

Little Popo émergea d'abord comme port, au cours de la seconde moitié du XVII siècle, $\mathrm{du}$ fait du rôle joué par les canots et leurs rameurs dans le commerce de la zone lagunaire. Le premier poste commercial européen (celui de la Royal African Company) date de 1688. La place vit ensuite se succéder les Européens à la recherche d'esclaves à l'époque de la traite atlantique. Cependant, par la faiblesse de ses liens avec l'intérieur du pays, elle n'atteignit jamais l'importance d'autres sites négriers comme Ouidah au Dahomey. Les premiers documents consignés dans le Grand Livre Lolamé (du nom du lieu où était situé le palais des Lawsons) concernent la seconde moitié du XIXe siècle, période au cours de laquelle la traite atlantique s'achève, faisant place au commerce dit " légitime ", c'est-à-dire principalement, dans la région, l'exportation d'huile de palme. Puis vint le temps de la pénétration coloniale, avec les Anglais d'abord, puis les Allemands et les Français (tous les documents du Grand Livre sont néanmoins rédigés uniquement en anglais). La temporalité couverte par ce document est donc vaste et particulièrement complexe. À ce jeu régional et international s'ajoutent les rivalités locales. Dans l'Aneho d'aujourd'hui comme dans celui d'hier, notent Jones et Sebald, histoire et politique sont étroitement mêlées. La ville dispose officiellement de deux chefs d'égal statut depuis 1961, l'un du clan Adjigo, l'autre des Lawsons. Certains documents du Grand Livre ont étésans nul doute écrits à des dates postérieures à l'époque décrite. Beaucoup, ajoutent les auteurs, l'ont été à des fins partisanes, bien qu'il soit difficile de savoir si ce qui y est dit est ou non totalement véridique. Ce qui nous renvoie à l'intérêt premier de cette publication, dont le contenu, comparé aux autres sources dont nous disposons sur la région et la période, permettra sans doute aux analystes les plus pointus d'en savoir finalement un peu plus.

4 En attendant, ce que l'on pourra regretter ici c'est le parti pris (qui se défend très bien, par ailleurs) de rester uniquement au plus près de la source et de n'en donner, finalement, qu'un éclairage historique, par des introductions permettant de contextualiser les faits narrés dans les documents. Uniques spécialistes de cette source en quelque sorte « inventée " par eux, Jones et Sebald auraient pourtant été les mieux à même de la mettre en perspective et de souligner des rapprochements possibles. Ce que Jones nous dit dans sa préface (p. xi), à propos de la maîtrise de l'anglais par un Africain du milieu du $\mathrm{XIX}^{\mathrm{e}}$ siècle et de son rapport complexe à l'écrit est extrêmement intéressant. On aurait aimé de plus nombreux passages cette sorte - et notamment que 
les raisons de ce passage à l'écrit et de cette volonté de garder une mémoire matérielle (et non plus seulement orale) de certains faits, soient, sinon explicitées, du moins présentées dans leur globalités, quitte à se limiter à de simples hypothèses de travail.

5 Ayant longuement étudié la correspondance familiale et commerciale des négociants et armateurs français qui vivaient à l'époque des événements relatés dans le Grand Livre Lolamé, j'ai été surpris par certains points communs avec les lettres du premier chapitre (1841-1853). Ces documents seraient relativement classiques en matière de correspondance commerciale européenne, à ce détail près que les lettres des Lawsons sont relativement courtes (un paragraphe ou deux) : on répond à un correspondant, on le remercie, on lui fait part ou on lui demande des informations, etc. Toutefois, on ne voit jamais une opération commerciale traitée dans le détail. Est-ce dû à un rapport particulier à l'écrit (considéré ici dans sa dimension cérémoniale et officielle plus que technique), à une manière différente de traiter les affaires commerciales (celles-ci devant alors demeurer plus orales), ou bien encore au contexte particulier de l'époque (passage de la traite, à laquelle les Lawsons ont participé, au commerce légitime qu'ils investissent tout en maintenant des relations avec des négriers notoires, notamment venus du Brésil, comme Jones et Sebald le soulignent) ? Avec les années 1854-1877, on voit les correspondances commerciales s'effacer au profit de documents cette fois-ci beaucoup plus longs et plus "politiques » : baptêmes, couronnements, énumération de la domesticité, questions judiciaires et militaires, etc. Il ne faut évidemment pas confondre ici récit de/et existence de/, car royauté et questions politiques préexistaient à leur entrée dans le Grand Livre. Il faut se demander pourquoi, à ce moment-là, il devient important pour les Lawsons d'en conserver une mémoire matérielle... et en anglais : volonté de manifester plus encore la puissance de la famille ; sentiment, au contraire (mais les deux hypothèses ne sont pas contradictoires), de perdre du terrain par rapport aux Européens, et donc désir de matérialiser l'influence exercée par les Lawsons pour et dans la «langue » (symbolique et non pas seulement vernaculaire) de leurs envahisseurs? Avec les années 1880, les préoccupations se font plus pragmatiques. On parle de traités, de disputes territoriales, le fonctionnement de la machine étatique passant alors au second plan.

6 Ces remarques ou interrogations ne font, au final, que révéler l'une des grandes qualités jusqu'ici non mentionnées de ce beau livre: celle consistant à nous faire réfléchir.

\section{AUTEURS}

\section{OLIVIER PÉTRÉ-GRENOUILLEAU}

Institut d'études politiques de Paris 